\title{
Increase of reactive oxygen species contributes to growth inhibition by fluconazole in Cryptococcus neoformans
}

\author{
Nadir Hani Dbouk, Madison Bailey Covington, Kenny Nguyen and Srikripa Chandrasekaran ${ }^{*}$ (D)
}

\begin{abstract}
Background: Cryptococcus neoformans, a basidiomycetous yeast, is a fungal pathogen that can colonize the lungs of humans causing pneumonia and fungal meningitis in severely immunocompromised individuals. Recent studies have implied that the antifungal drug fluconazole (FLC) can induce oxidative stress in C. neoformans by increasing the production of reactive oxygen species (ROS), as presence of the antioxidant ascorbic acid (AA) could reverse the inhibitory effects of FLC on C. neoformans. However, in Candida albicans, AA has been shown to stimulate the expression of genes essential for ergosterol biosynthesis. Hence, the contribution of ROS in FLC-mediated growth inhibition remains unclear.

Results: In order to determine whether counteracting ROS generated by FLC in C. neoformans can contribute to diminishing inhibitory effects of FLC, we tested three other antioxidants in addition to AA, namely, pyrrolidine dithiocarbamate (PDTC), retinoic acid (RA), and glutathione (GSH). Our data confirm that there is an increase in ROS in the presence of FLC in C. neoformans. Importantly, all four antioxidants reversed FLC-mediated growth inhibition of $C$. neoformans to various extents. We further verified the involvement of increased ROS in FLC-mediated growth inhibition by determining that ROS-scavenging proteins, metallothioneins (CMT1 and CMT2), contribute to growth recovery by PDTC and AA during treatment with FLC.
\end{abstract}

Conclusion: Our study suggests that ROS contributes to FLC-mediated growth inhibition and points to a complex nature of antioxidant-mediated growth rescue in the presence of FLC.

Keywords: Antifungal treatment, Antioxidants, ROS, Metallothionein

\section{Background}

Eukaryotic pathogens, including pathogenic fungi are an important cause of death in immunocompromised patients and can colonize immunocompetent individuals [1]. Cryptococcal meningitis caused by Cryptococcus neoformans is the leading cause of fungal central nervous system infection in the world, especially among persons suffering from HIV/AIDS [2, 3]. According to CDC reports, annually one million global cases of cryptococcal infections occur, accounting for up to 600,000 mortalities and about one-third of all AIDS-associated deaths. Despite the severity of cryptococcosis, unfortunately current treatments for cryptococcal infections are inadequate. A main barrier to establishment of an effective

\footnotetext{
* Correspondence: Srikripa.Chandrasekaran@furman.edu

Department of Biology, Furman University, Greenville, SC, USA
}

antifungal drug therapy is increased drug resistance in fungi [4-6].

Compared to other anti-cryptococcal drugs, fluconazole (FLC) is the more affordable and less toxic alternative, which is most commonly prescribed in geographic locations where cryptococcosis is most prevalent $[7,8]$. FLC is the drug of choice for moderate pulmonary infections. For central nervous system infections, a combination of more expensive fungicidal drugs amphotericin $B$ and flucytosine are administered [9, 10]; however, the combination of these two drugs produces more toxic side effects for the host.

A well-established mechanism of action of FLC is the inhibition of Erg11, which is one of the key enzymes participating in the synthesis of ergosterol, an important component of the plasma membrane [11]. One factor that contributes to failure of FLC-based therapy is the

(c) The Author(s). 2019 Open Access This article is distributed under the terms of the Creative Commons Attribution 4.0 International License (http://creativecommons.org/licenses/by/4.0/), which permits unrestricted use, distribution, and 
development of drug resistance. FLC resistance in $C$. neoformans occurs primarily via development of aneuploid cells with elevated levels of Erg11, which prevents diminishment of ergosterol [12]. Other causes for FLC resistance in pathogenic fungi include accumulation of mutations in ERG11 [13] and via drug efflux pumps [14, 15]. Importantly, the mechanisms through which FLC leads to formation of aneuploid and FLC resistant cells remain largely uncharacterized.

While diminishment of ergosterol is a well-documented cause of FLC-mediated growth inhibition of $C$. neoformans, additional possible effects of FLC on C. neoformans cells have been proposed. FLC treatment has been shown to cause an increase in reactive oxygen species (ROS) in Candida albicans [16-18] and most recently in C. neoformans [19]. ROS are molecules with unpaired, highly reactive electrons called free radicals, generated during basic cellular processes, or due to external stress-inducing conditions, including environmental pollutants, foreign compounds such as drugs or chemicals, and exposure to X-rays [20]. Free radicals are highly reactive and unstable and excessive amounts of ROS are known to cause cell damage and trigger apoptosis. Generation of high amounts of free radicals can be harmful to biological macromolecules, as it can cause modification of DNA bases [21], lipid peroxidation, and protein carbonylation [22] leading to damage due to oxidative stress. Some examples of ROS include hydroxyl radicals, hydroxide anion radicals, singlet oxygen, hydrogen peroxide, hypochlorite, nitric oxide radicals, and peroxynitrite radicals. FLC-mediated increase in ROS could contribute towards oxidative stress in $C$. neoformans. Consistent with FLC-triggered ROS contributing to growth inhibition, co-treatment of $C$. neoformans cells with FLC and the antioxidant ascorbic acid (AA) was shown to partially rescue $C$. neoformans cells from FLC-mediated growth inhibition [19]. Similarly, co-treatment of C. albicans cells with the anti-fungal drug miconazole and a synthetic antioxidant, pyrrolidine dithiocarbamate (PDTC), has been shown to increase the Minimal Inhibitory Concentration (MIC) of miconazole [18]. These studies suggest an additional effect of anti-fungal azole drugs on pathogenic fungi, which is inducing oxidative stress via an increase in ROS content.

Interestingly, treatment of $C$. albicans with AA has been shown to increase expression of the gene UPC2, which is involved in regulating ergosterol biosynthesis $[23,24]$. This finding suggests that AA might be functioning indirectly to regulate ergosterol levels, which is by counteracting FLC-mediated inhibition of ergosterol biosynthesis. Hence, whether ROS increase triggered by FLC contributes to growth inhibition elicited by FLC remains unclear.

The metal copper has been shown to be essential for virulence of $C$. neoformans [25]. Lack of a copper transporter, CTR4, led to reduced virulence in cryptococcosis models in mice [26]. During infection by C. neoformans, copper acquisition and increased copper levels is essential for melanin formation, which confers virulence to $C$. neoformans [27]. While elevated copper is essential during infection, increased copper can be toxic as it contributes to increased production of ROS, due to its participation in oxidation and reduction reactions [28]. To counteract harmful effects of copper, C. neoformans increases expression of metallothionein genes, CMT1 and CMT2, which bind to and sequester copper [29]. Previous studies have shown that $C$. neoformans mutants lacking metallothionein genes exhibit attenuated virulence [30] and show an increased sensitivity to FLC [19]. These findings suggest that $\mathrm{Cmt} 1$ and $\mathrm{Cmt} 2$ proteins allow for reversal of some of the harmful effects of ROS generated in the presence of FLC.

The purpose of this study was to perform a more rigorous test to determine if ROS plays a role in influencing sensitivity to FLC in C. neoformans. In order to determine whether it is the antioxidant properties of AA that caused rescue of $C$. neoformans growth inhibition, we tested three alternative known antioxidants for their ability to reverse the effects of FLC on the wild type as well as on metallothionein deficient mutants. Our data suggest that treatment with FLC leads to increase of ROS and this oxidative stress may further contribute to FLC-mediated growth inhibition. Furthermore, this study suggests that lowering ROS is not the only contributing factor to the antioxidant-mediated growth rescue and points to the complex nature of the physiological effects of FLC.

\section{Results}

We wished to determine whether antioxidants with diverse chemical structures and modes of action could alleviate FLC-mediated growth inhibition of C. neoformans. In addition to AA that has been previously shown to reduce growth inhibition in the presence of FLC in C. neoformans [19], we tested three chemically unrelated antioxidants: pyrrolidinedithiocarbamate (PDTC), retinoic acid (RA), and a reduced form of glutathione (GSH). The concentrations of AA, PDTC, and GSH were established based on previous studies $[18,19,31]$. The concentration of RA was established as the smallest concentration that rescued growth of C. neoformans cells in the presence of hydrogen peroxide (as later indicated in Fig. 3a). Growth of cells on plates with media supplemented with the respective amounts of the antioxidants and lacking FLC was not inhibited as compared to the control YPD media (as indicated in Fig. 2b). As shown in Fig. 1a, in the presence of $32 \mu \mathrm{g} / \mathrm{ml} \mathrm{FLC,} \mathrm{cell}$ growth was significantly inhibited, although single colonies of cells that were likely resistant to FLC were observed. Cotreatment of cells with both FLC and any of the four antioxidants led to rescue of growth. While RA, AA and PDTC 


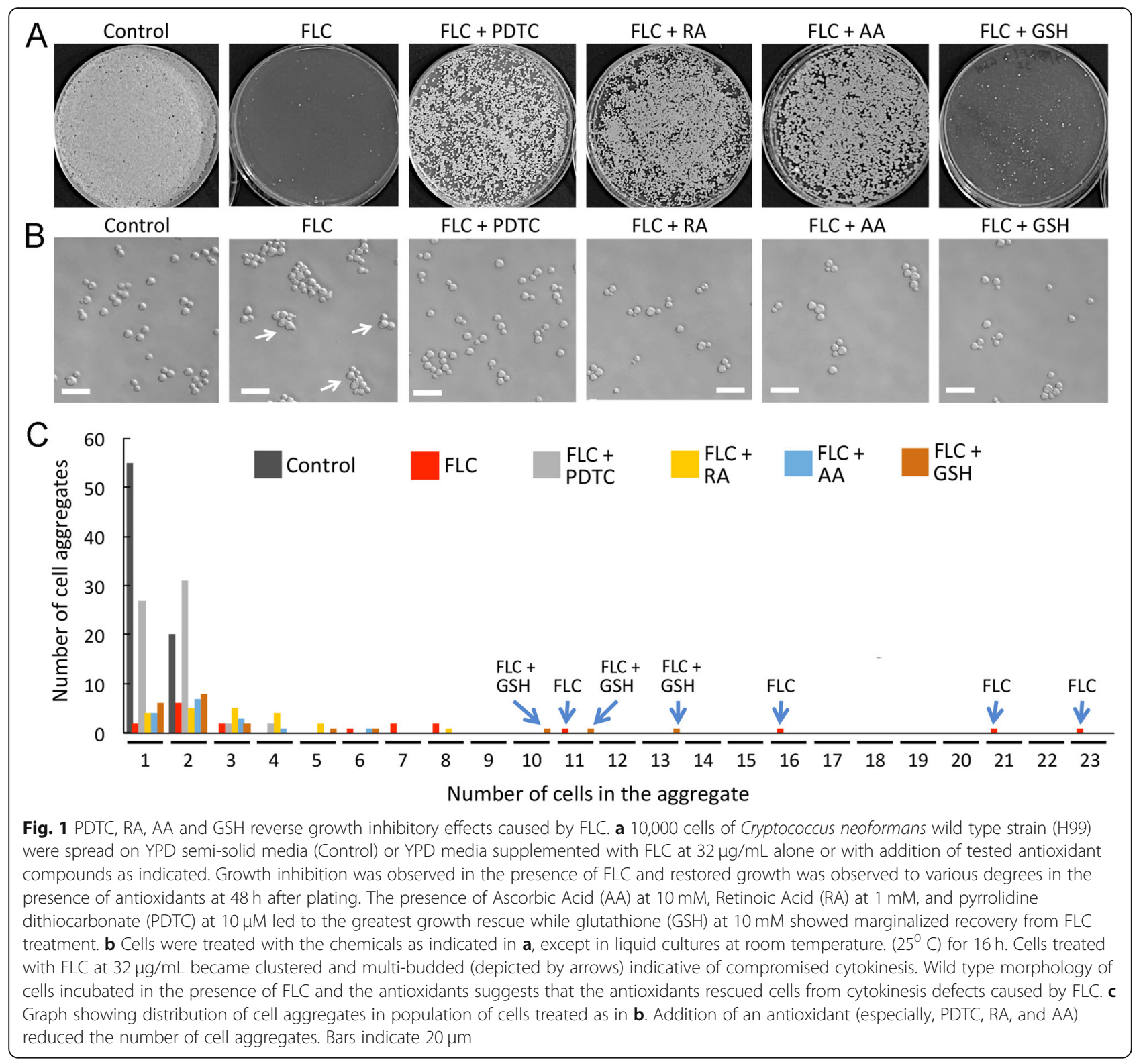

showed a robust reversal of FLC-mediated growth inhibition, GSH showed only a modest rescue of growth.

It has been previously demonstrated that treatment of $C$. neoformans with FLC causes cytokinesis defects, visible as a multi-budded phenotype [32]. In order to determine whether the antioxidants can reverse the multi-budded phenotype resulting from FLC treatment, we treated $C$. neoformans cells for $16 \mathrm{~h}$ with either $32 \mu \mathrm{g} / \mathrm{ml}$ FLC alone or FLC and an antioxidant (either RA at $1 \mathrm{mM}, \mathrm{AA}$ at $10 \mathrm{mM}$, PDTC at $10 \mu \mathrm{M}$, or GSH at $10 \mathrm{mM}$ ). As shown in Fig. 1b, cells treated with FLC alone exhibited a multi-budded phenotype indicative of a cytokinesis defect, consistent with previous studies (Fig. 1b, arrows). When cells were cotreated with FLC and either AA, RA, PDTC, or GSH, cell morphology was similar to that of the control sample and no significant multi-budded phenotype was observed (Fig. 1b). Thus, the ability of tested antioxidants to rescue C. neoformans cells from FLC-mediated growth inhibition correlated with the ability of each of the antioxidants to reduce the multi-budded morphology of cells resulting from FLC treatment. In addition to morphological defects, C. neoformans cells exhibited clumping phenotype in the presence of $32 \mu \mathrm{g} / \mathrm{ml}$ FLC. As represented in Fig. 1c, cell aggregates were drastically reduced in the presence of AA, RA, PDTC, or GSH. These findings suggest that the multibudded phenotype and cell aggregation resulting from FLC treatment is at least partially caused by the increase of ROS, based on the ability of various antioxidants to reverse these 
phenotypes. Interestingly, while GSH could rescue FLCinduced morphological growth defects, as shown in Fig. 1b, co-treatment of cells with FLC at $32 \mu \mathrm{g} / \mathrm{ml}$ and GSH at 10 $\mathrm{mM}$ led to only a modest rescue of growth, in contrast to addition of AA, RA, or PDTC, as shown in Fig. 1a. These findings suggest that the tested anti-oxidants reverse the effects of FLC through mechanisms that may not be identical.

Copper levels are increased during C. neoformans infection and the increased copper aids $C$. neoformans virulence, while also contributing to an increase in ROS $[25,30]$. Metallothionein proteins ( $\mathrm{Cmt} 1$ and $\mathrm{Cmt} 2)$, whose levels increase in response to copper, have been implicated in lowering ROS by sequestering heavy metals such as copper [33]. Since it has been shown that C. neoformans mutants lacking metallothioneins are more sensitive to FLC [19], we tested whether metallothioneins are required for antioxidant-mediated growth rescue in the presence of FLC. We carried out a serial dilution assay where we spotted wild type, $\operatorname{cmt} 1 \Delta$, cmt $2 \Delta$, or $c m t 1 / 2 \Delta$ mutants as a series of 10 -fold diluted cell cultures. As shown in Fig. 2a, all the strains grew equally well on a control YPD medium, while on YPD medium supplemented with FLC $(32 \mu \mathrm{g} / \mathrm{ml})$ all strains were significantly inhibited. However, growth of wild type was robustly rescued when cells were grown on YPD medium supplemented with FLC and RA (Fig. 2a), Growth rescue of the wild type was less evident in the presence of AA, or PDTC, and it was minimal in the presence of GSH. Compared to wild type cells, growth of the metallothionein mutants could not be rescued to the same extent when grown in YPD medium supplemented with FLC and PDTC, AA, RA, or GSH. Consistently, both wild type and $c m t$ mutant strains showed maximum rescue from FLC inhibition by RA (Fig. 2a). This finding suggests that metallothionein proteins are necessary for the effective growth rescue by antioxidants when cells are treated with FLC.

To further test whether treatment of wild type vs metallothionein mutant strains (cmt1,$c m t 2 \Delta$ and $c m t 1 /$ $2 \Delta$ ) results in change in sensitivity to FLC, we carried out a disc diffusion assay, which allowed us to compare the effects of increasing amounts of FLC. We plated $2 \times$ $10^{6}$ cells of either wild type (H99) or metallothionein mutants on YPD semisolid medium and we placed on the surface of the medium a control disc and 3 discs containing 25, 50, or $100 \mu \mathrm{g}$ of FLC. As shown in Fig. 2b, all three metallothionein mutant strains exhibited higher sensitivity to FLC, as indicated by larger zones of inhibition surrounding the discs containing FLC, as compared to wild type strain. We also utilized the disc diffusion assays to examine the effects of antioxidants on the sensitivity of metallothionein mutant strains to FLC. As indicated in Fig. 2b, and graphed in Fig. 2c (based on discs containing 100 micrograms of FLC), wild type strain (H99) exhibited a significant recovery from FLCmediated growth inhibition in the presence of AA, RA, PDTC, and GSH with PDTC appearing as most potent. In contrast, for all three metallothionein mutants, cmt $1 \Delta$, $c m t 2 \Delta$, or $c m t 1 / 2 \Delta$, the recovery from FLC mediated inhibition in the presence of PDTC was less significant as compared to the wild type (Fig. 2c). The cmt1/2A double mutant was unable to significantly recover from FLCmediated growth inhibition in the presence of AA (Fig. 2b, c). In the presence of RA and GSH, all metallothionein mutants could recover from growth inhibition caused by FLC (Fig. 2b, c). These findings suggest that the antioxidants tested may function in different ways to reverse growth inhibition caused by FLC in C. neoformans. PDTC-mediated growth recovery of $C$. neoformans and to a lesser extent also AA-mediated growth recovery of $C$. neoformans, in the presence of FLC, may require expression of metallothioneins, while for RA and GSH to exert their effects metallothioneins activity may not be critical.

Our data suggested that the degree to which the antioxidants reversed the inhibition by FLC was unequal. One explanation of these differences may be the ability of each antioxidant to reduce ROS at applied concentrations. To test this possibility, we examined the capacity of each of the antioxidants to reduce ROS in C. neoformans. First, we utilized hydrogen peroxide, which is a well-established ROS-generating agent that induces oxidative stress in $C$. neformans cells, as determined by increased expression of enzymatic antioxidants, including CAT1, CAT3, and TRR1 [34]. We performed a growth spot assay with the wild type (H99) strain in either YPD media plates, YPD supplemented with $3 \mathrm{mM}$ hydrogen peroxide, or YPD supplemented with $3 \mathrm{mM}$ hydrogen peroxide and one of the four antioxidants (AA, RA, PDTC, or GSH). As shown in Fig. 3a, in the presence of $3 \mathrm{mM}$ hydrogen peroxide, growth of cells was dramatically reduced. Strikingly, the presence of $10 \mathrm{mM} \mathrm{GSH}$ or $10 \mathrm{mM}$ AA led to a complete rescue of growth from the inhibitory effects of hydrogen peroxide, which indicates that AA and GSH are potent antioxidants in C. neoformans. RA and PDTC also rescued growth inhibition by hydrogen peroxide, but not to the same extent as GSH or AA (Fig. 3a). These results were further confirmed by the disc diffusion assay, in which wild type cells were plated on either YPD or YPD media supplemented with an antioxidant (AA, RA, GSH or PDTC) and exposed to a control disc and 3 discs with increasing concentrations of hydrogen peroxide $(25,50$, or $100 \mathrm{mM})$. As shown in Fig. $3 \mathrm{~b}$, in the absence of an antioxidant, distinct zones of inhibition were formed that increased in diameter, as the concentration of hydrogen peroxide increased. When YPD was supplemented with the antioxidants GSH and AA, the diameters of the zones of inhibition at all the concentrations of hydrogen 


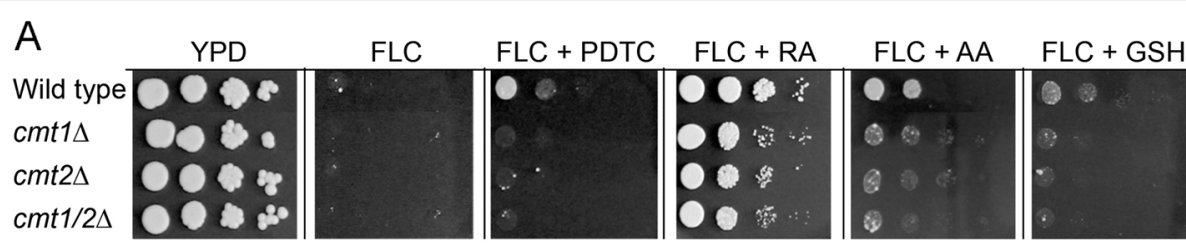

$\mathrm{B}$

Control PDTC

RA

AA

GSH

Wild type
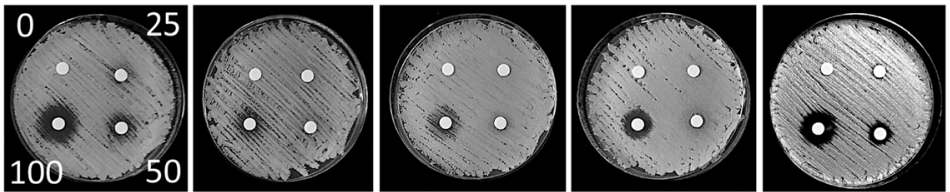

$c m t 1 \Delta$
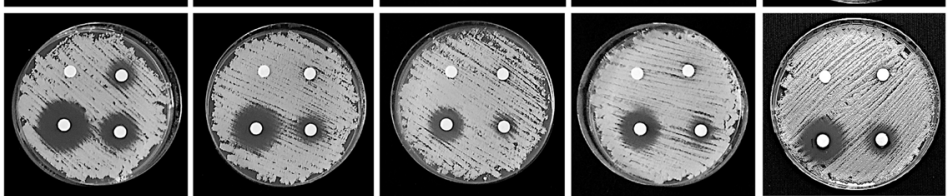

$c m t 2 \Delta$
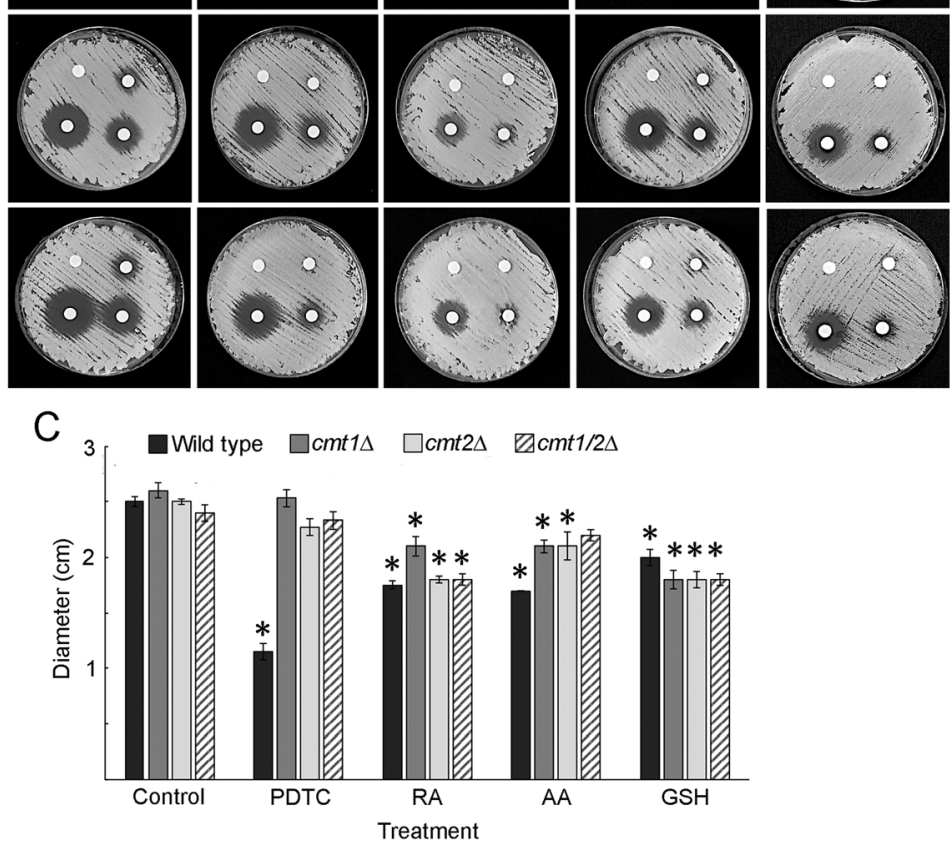

Fig. 2 Metallothioneins contribute to antioxidant-mediated growth rescue in the presence of FLC. a Growth spot assay of wild type (H99), and the $\mathrm{cmt} 1 \Delta, \mathrm{cmt} 2 \Delta, \mathrm{cmt1} / 2 \Delta$ mutants on YPD, or YPD supplemented with FLC $(32 \mu \mathrm{g} / \mathrm{mL})$ or FLC and an antioxidant (10 mM PDTC, $1 \mathrm{mM} \mathrm{RA,} 10$ mM AA, $10 \mathrm{mM} \mathrm{GSH).} \mathbf{b} 2 \times 10^{6}$ of wild type cells (H99) or metallothionein mutants were spread on YPD semi-solid media or YPD media

supplemented with antioxidants at concentrations as described in a. Discs containing increasing amounts of FLC $(25,50$, or $100 \mu \mathrm{g})$ were placed on media and the growth inhibition zones were examined after 2 days of incubation at $25^{\circ} \mathrm{C}$. $\mathbf{c}$ Graph based on quantification of the results of the experiment described in $\mathbf{b}$ (based on three replicates). Error bars indicate standard deviation. Stars indicate significant growth rescue by the antioxidant as compared to the control treatment $(p<0.05)$

peroxide tested were smaller. However, presence of PDTC or RA in the YPD media did not lead to the reduction of the zones of inhibition in the presence of hydrogen peroxide, indicating that AA and GSH were more effective antioxidants to alleviate the effects of hydrogen peroxide in $C$. neoformans when compared to PDTC and RA. Thus, our results suggest that although all the antioxidants could rescue growth inhibition in the presence of hydrogen peroxide, GSH and AA are more potent antioxidants than $\mathrm{RA}$ and PDTC in C. neoformans.

To further test whether addition of the antioxidants (RA, AA, PDTC, or GSH) could reverse oxidative stress that is present during FLC treatment, we carried out a fluorescent assay to detect Reactive Oxygen Species (ROS). We used a ROS indicator, 4-Amino-5-methylamino-2',7'-difluorofluorescein diacetate $\left(\mathrm{H}_{2} \mathrm{DCFDA}\right)$, which binds to free 


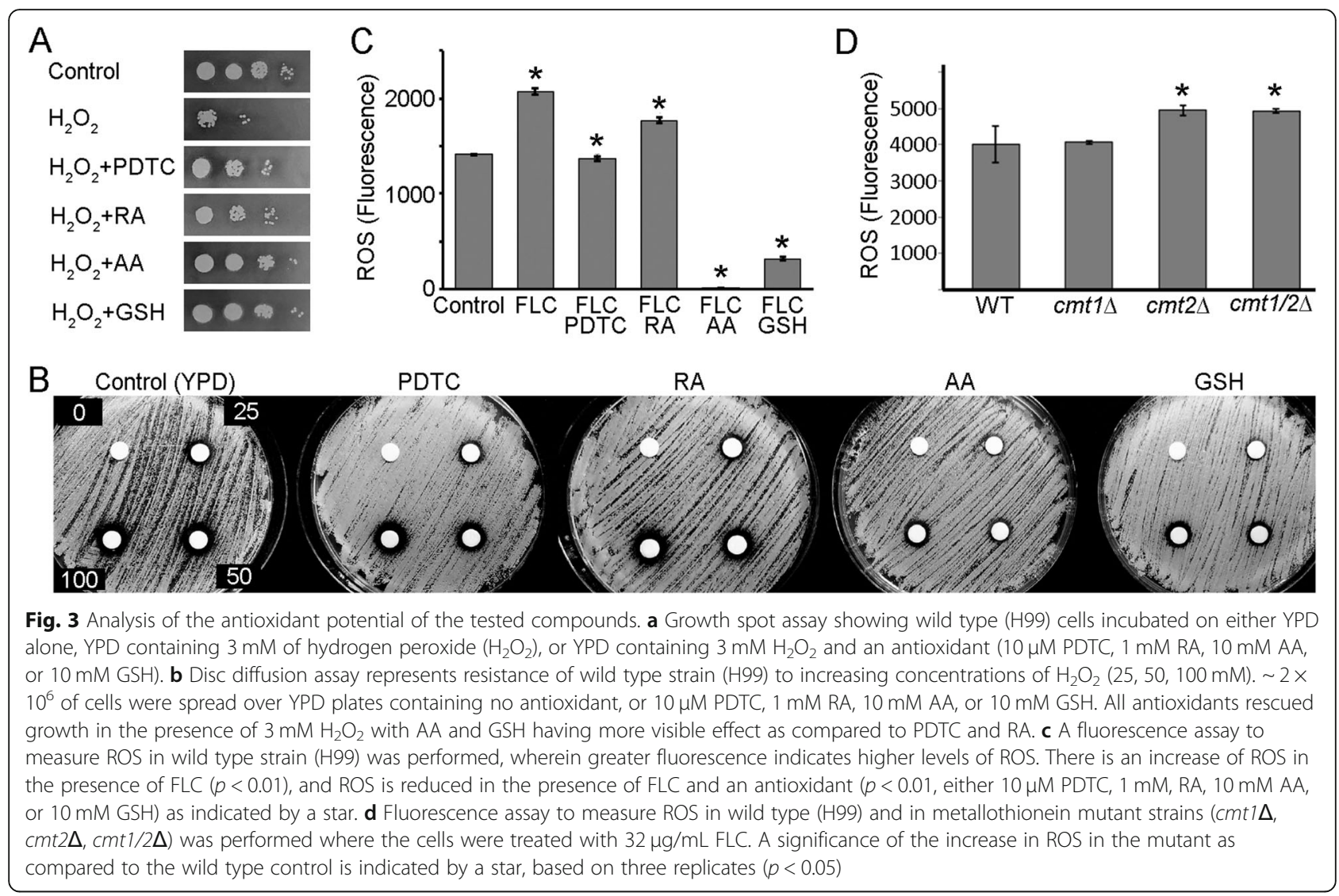

radicals within the cell and can be detected as a fluorescent signal at an excitation wavelength of $495 \mathrm{~nm}$ and emission wavelength of $529 \mathrm{~nm}$, as used in a recent study [19]. As shown in Fig. 3c, treatment with FLC led to elevated ROS and co-treatment with either GSH, AA, RA, or PDTC significantly lowered levels of ROS. While all antioxidants lowered ROS generated in the presence of FLC, GSH and AA led to the most significant decrease in ROS, while the effect of RA and PDTC was less pronounced. Furthermore, GSH and AA appeared to lower ROS significantly below the endogenous levels, while PDTC and RA specifically led to a decrease of ROS generated upon addition of FLC.

Based on data implicating metallothioneins in counteracting the inhibitory effects of FLC, we hypothesized that FLC treatment may lead to a more extensive accumulation of ROS in the metallothionein mutants as compared to the wild type. In order to test whether the ROS generated by wild type (H99) cells was lower compared to metallothionein mutants (either $c m t 1 \Delta, c m t 2 \Delta$, or $c m t 1 / 2 \Delta$ ), we carried out fluorescence measurements using the dye $\mathrm{H}_{2}$ DCFDA. As shown in Fig. 3d, the overall ROS was significantly higher in $c m t 2 \Delta$ and the $c m t 1 / 2 \Delta$ double mutants as compared to the wild type. These results suggest that the CMT2 metallothionein protein contributes to lowering ROS in C. neoformans during FLC treatment.

\section{Discussion}

The main aim of this study was to determine whether treatment with various antioxidants could reverse growth defects and morphological defects caused by FLC treatment in C. neoformans. Previous studies have implicated FLC to be involved in generation of ROS in C. albicans [16-18] and in C. neoformans [19]. It has been shown that AA can rescue growth inhibition caused by FLC in C. neoformans [19]. It has been demonstrated that addition of AA leads to induction of ERG11 gene expression to allow for increased ergosterol production and this process is mediated by a transcription factor Upc2 [23, 24]. Hence, how AA reduces growth inhibition caused by FLC remains unclear. The effect of FLC on ROS in C. neoformans shown in the study by Peng et al. appears insignificant compared to the increase of ROS caused by another antifungal drug, amphotericin B [19, 35]. Another azole drug, itraconazole, led to ROS formation and lipid peroxidation in a sibling species $C$. gattii in the early stages of the treatment; this did not occur with fluconazole [36]. Therefore, the contribution of ROS in C. neoformans during FLC treatment remains unclear. We reasoned that if antioxidants indeed reverse oxidative stress generated by ROS, then various antioxidants should rescue growth inhibition of $C$. neoformans in the presence of FLC. 
While we found that all tested antioxidants (AA, RA, PDTC, and GSH) could rescue growth inhibited by FLC (using growth assays, spot assays and disc diffusion assays to assess sensitivity to FLC), the rescue was not uniform. If we consider a measure of growth rescue in the presence of hydrogen peroxide as an indicator of the antioxidant potential, AA and GSH were the most efficient antioxidants in C. neoformans, while PDTC and RA were less effective as antioxidants compared to AA and GSH. Interestingly, while GSH was one of the more potent antioxidants, based on the ability of GSH to lower ROS caused by hydrogen peroxide and FLC, GSH only moderately rescued growth inhibition by FLC. This suggests that the antioxidants may be counteracting specific species of ROS not always matching ROS type that is generated during oxidative stress in the presence of FLC. A non-exclusive possibility is that the effects of the antioxidants also involve changes in the expression of ergosterol pathway genes in addition to lowering ROS and collectively contribute to the survival in the presence of FLC. Future studies that would examine ergosterol levels and measure global gene expression in the presence of FLC and specific antioxidants will help to resolve these uncertainties.

Interestingly, each of the antioxidants tested could rescue morphological growth defects caused by FLC. $C$. neoformans wild type (H99) strain treated with (FLC) at $32 \mu \mathrm{g} / \mathrm{mL}$ displayed multi-budded phenotype most likely due to inability to perform cytokinesis. Our study revealed that all the antioxidants tested (AA, RA, PDTC, and GSH) can rescue cells from cytokinesis defects caused by FLC, but not all antioxidants could rescue growth inhibition due to FLC to the same extent. This result suggests that while cytokinesis defect may contribute to growth defect in FLC-treated cells, eliminating this aberration is not sufficient to restore growth in the presence of FLC.

Another evidence that FLC is contributing to an increase in ROS in C. neoformans is the involvement of the metallothionein genes CMT1 and CMT2 in resisting the inhibitory effects of FLC. Metallothionein proteins are essential for sequestering copper levels, which are upregulated during infection by C. neoformans [29]. Increased copper levels can induce ROS, hence during infection by $C$. neoformans, expression of CMT genes is crucial. Previous studies have shown that mutants of $C$. neoformans, lacking $\mathrm{cm} t$ genes are more sensitive to FLC treatment [19]. Our results suggest that cells lacking Cmt2 or both Cmt1 and Cmt2 proteins are more sensitive to FLC. We also find that Cmt mutants are compromised in their ability to recover $C$. neoformans cells from FLC treatment when antioxidants are added. Interestingly, the extent to which the mutants could be rescued when co-treated with FLC and an antioxidant varied depending on the type of antioxidant. We find that Cmt proteins play an important role in PDTC-based growth rescue in FLC-treated cells. This further suggests that these antioxidants act through various molecular mechanisms to facilitate rescue from FLC-mediated cell growth inhibition. Future studies should determine the effects of the antioxidants on gene expression in cells treated with FLC. Including Cmt mutants in transcriptional profiling of $C$. neoformans during various treatments would shed light on molecular mechanisms responsible for FLC resistance in C. neoformans.

The antioxidants we have tested in this study have been shown to reverse damage caused by many types of free radicals. PDTC has been shown to reverse oxidative damage and carbonylation of proteins by reversing $\mathrm{HOCl}-$ mediated oxidative stress [37]. RA has been implicated in hydroxyl radical and lipid peroxide scavenging [38]. AA has been shown to reverse oxidative stress mainly caused by oxygen free radicals $[39,40]$. GSH has been implied in reversing oxidative stress generated by hydrogen peroxide [41] and lipid peroxides [42]. Previous studies using $C$. glabrata as a model have suggested that FLC causes an increase in singlet oxygen and peroxide radicals and can cause DNA damage and treatment of Candida with FLC increased activity of enzymatic antioxidants, namely superoxide dismutase (SOD) and glutathione peroxidase (GPx) [16]. It is possible that reversal of ROS and growth defects in the presence of AA and GSH in C. neoformans is due to the quenching of singlet oxygen species and hydrogen peroxide damage caused by FLC. In addition to DNA damage, it is possible that protein oxidation and carbonylation could be increased in the presence of FLC, which would explain the role played by PDTC in reversing FLC damage in C. neoformans. FLC has been shown to be more potent in Candida species strains defective in superoxide dismutase and catalase activity [43]. Hence RA could have restored FLC-mediated growth inhibition by regulated SOD levels in the presence of FLC. Further investigations should determine what specific forms of free radicals are upregulated in the presence of FLC and the extent of DNA and protein damage that could be caused in the presence of FLC.

\section{Conclusions}

In summary, we conclude that one of the effects of FLC treatment in C. neoformans is an increase in ROS. Furthermore, addition of antioxidants can partially rescue growth of C. neoformans in the presence of FLC. However, our results point to a complex nature of the effects of the antioxidants and suggest that various mechanisms contribute to the antioxidant-mediated growth rescue. The significance of this study is in understanding environmental conditions that can cause rescue of growth of C. neoformans in the presence of FLC and potentially development of resistance to FLC. While formation of 
aneuploid cells is associated with FLC resistance, recent studies are revealing that counteracting ROS caused by FLC in fungi could also contribute to resisting FLC mode of action. Understanding how individual antioxidants could reverse ROS generated by FLC and tying their effects to transcriptional profiling of genes that get altered during co-treatment with FLC and antioxidants would uncover molecular mechanisms that potentially lead to FLC resistance in C. neoformans and other pathogenic fungi.

\section{Methods}

\section{Reagents used}

Ascorbic Acid or AA (Fisher Scientific, Cat No A61-25, CAS 5081-7) was prepared from a stock of $1 \mathrm{M}$, and used at $10 \mathrm{mM}$. A reduced form of glutathione or GSH (Alfa Aesar, Cat No AAJ6216606, CAS 70-18-8) was prepared from a stock of $0.5 \mathrm{M}$, and used at $10 \mathrm{mM}$. Pyrrolidinedithiocarbamate or PDTC (Cayman Chemicals, Cat No 20713, CAS 5108-96-3) was prepared from a stock of 10 $\mathrm{mM}$, and used at $10 \mu \mathrm{M}$. Retinoic Acid or RA (Cayman Chemical, Cat No 11017, CAS 302-79-4) was prepared from a stock of $100 \mathrm{mM}$ (dissolved in dimethyl sulfoxide (DMSO)), and used at $1 \mathrm{mM}$. The fluorescent dye for ROS assays, 4-Amino-5-methylamino-2',7' -difluorofluorescein diacetate $\left(\mathrm{H}_{2} \mathrm{DCFDA}\right)$ (Sigma, Cat No D6883, CAS 4091-99-0), was dissolved in DMSO at a stock concentration of $100 \mathrm{mM}$ and used at $10 \mu \mathrm{M}$. Fluconazole (Cayman Chemical, Cat No 11594, CAS 86386-73-4) was dissolved in DMSO as a $50 \mathrm{mg} / \mathrm{ml}$ stock and used at $32 \mu \mathrm{g} / \mathrm{ml}$. Hydrogen peroxide (Cat No H325-100) was obtained from Fisher Scientific.

\section{Strains and media}

Cryptococcus neoformans var. grubii wild type (strain H99 Stud) is the derivative of the original strain isolated in 1978 by John Perfect at Duke University (ATCC 208821) that has been passaged through a rabbit at that time. The

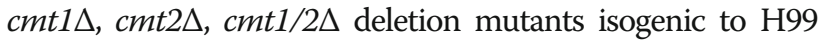
(CMT1, CNAG_05549; CMT2, CNAG_00306) were kindly provided by the laboratory of Dr. Lukasz Kozubowski, Clemson University (the metallothionein mutants were originally obtained from Dr. Dennis J. Thiele, Duke University).

Cells were grown on YPD media: (1\% yeast extract, $2 \%$ peptone, $2 \%$ dextrose, $2 \%$ agar), supplemented with chemicals as indicated in the text.

\section{Fluconazole sensitivity plate and spot growth assays}

Either wild type, $c m t 1 \Delta, c m t 2 \Delta$ or $c m t 1 / 2 \Delta$ were grown in liquid YPD broth overnight for $16 \mathrm{~h}$. All strains were diluted to an Optical Density of $\mathrm{OD}_{600}=0.1$ and refreshed in YPD liquid media for $4 \mathrm{~h}$ and then counted using a Neubauer Hemocytometer. For growth assays, 10,000 cells in exponential growth phase were spread onto plates containing either YPD media alone, YPD plus $32 \mu \mathrm{g} / \mu \mathrm{L}$ FLC, and YPD plus $32 \mu \mathrm{g} / \mu \mathrm{L}$ FLC and an antioxidant, namely, $10 \mu \mathrm{M}$ PDTC, $1 \mathrm{mM}$ RA, $10 \mathrm{mM} \mathrm{AA}$, or $10 \mathrm{mM}$ GSH. Spot growth assays were performed with a 10-fold serial dilution of cells such that $2 \mu \mathrm{L}$ contained either $10^{4}$, $10^{3}, 10^{2}$, or 10 cells and were carefully spotted onto YPD plates alone, YPD plus $32 \mu \mathrm{g} / \mu \mathrm{L}$ FLC, or YPD plus FLC and individual antioxidants, as described above. For both growth assays and spot assays, cells grew for $48 \mathrm{~h}$ at $25^{\circ} \mathrm{C}$ before recording the data.

\section{Fluorescence assay to detect ROS}

Cells were grown overnight at room temperature in $2 \mathrm{ml}$ liquid YPD medium with constant agitation, diluted to an Optical Density $\mathrm{OD}_{600}=0.1$, and grown for an additional $4 \mathrm{~h}$. Subsequently, the culture was diluted to 10 , 000 cells $/ \mathrm{ml}$ and the cultures were either grown as no treatment control, treated with either $32 \mu \mathrm{g} / \mathrm{ml} \mathrm{FLC,} \mathrm{or}$ $32 \mu \mathrm{g} / \mathrm{ml} \mathrm{FLC} \mathrm{and} \mathrm{an} \mathrm{antioxidant} \mathrm{(either} 10 \mu \mathrm{M}$ PDTC, $1 \mathrm{mM}, \mathrm{RA}, 10 \mathrm{mM}$ AA, or $10 \mathrm{mM} \mathrm{GSH}$ ) for $12 \mathrm{~h}$. To detect ROS, $10 \mu \mathrm{M}$ of a fluorescent dye, $\mathrm{H}_{2}$ DCFDA, was added to each of the samples and incubated for $1 \mathrm{~h}$ in the dark at $25^{\circ} \mathrm{C}$. A control set of each of the samples were incubated without the fluorescent dye. $250 \mu \mathrm{L}$ of the sample were added to each well of a 96-well microplate. ROS was measured as fluorescence emitted by the fluorescent dye, $\mathrm{H}_{2}$ DCFDA, at an excitation wavelength of $485 \mathrm{~nm}$ and an emission wavelength of $535 \mathrm{~nm}$. The fluorescence reading was measured and recorded as Relative Fluorescence Units (RFU). From each reading of the sample treated with $\mathrm{H}_{2}$ DCFDA the reading obtained from the sample without addition of $\mathrm{H}_{2}$ DCFDA was subtracted. Each treatment was made in triplicate. All data points were computed using multifactorial ANOVA and Tukey's HSD post hoc test.

\section{Disk diffusion assay}

C. neoformans strains (wild type H99, or mutants, cmt $1 \Delta$, cmt $2 \Delta$, or cmt1/2A) were grown in $2 \mathrm{ml}$ of YPD liquid broth overnight, for $16 \mathrm{~h}$, diluted to an $\mathrm{OD}_{600}=$ 0.1 and refreshed for $4 \mathrm{~h}$. Each strain was counted using a hemocytometer and $\sim 2 \times 10^{6}$ cells were plated onto YPD semi-solid media plates containing either no antioxidant (control), AA (10 mM), RA (1 mM), PDTC $(10 \mu \mathrm{M})$ or GSH $(10 \mathrm{mM})$, and spread with sterile Dynarex cotton tipped applicators at opposing $90^{\circ}$ angles. The plates were left to dry before application of cotton disks. After $10 \mathrm{~min}$ of drying, $6.6 \mathrm{~mm}$ cotton disks were lightly placed perpendicular on top of the YPD medium as to not break the surface of the gel. Depending on the experiment, either increasing amounts of 25, 50 and 100 micrograms of FLC, or increasing concentrations of 25,50 , and $100 \mathrm{mM}$ hydrogen peroxide were added to the top end of the disk in order for the FLC or hydrogen peroxide to diffuse 
throughout the area surrounding the disc. Finally, the discs were laid flush onto the medium equidistant from one another. The cells grew for $48 \mathrm{~h}$ at $25^{\circ} \mathrm{C}$ and all treatments were done in triplicate. Each zone of inhibition was measured and the results from each of the three replicate experiments were averaged. A multifactorial ANOVA along with a Tukey's HSD post hoc test was used to indicate significance.

\section{Microscopy}

Differential interference contrast (DIC) microscopy was used to study $C$. neoformans cell morphology under various conditions. C. neoformans cells were grown for $16 \mathrm{~h}$ at $25^{\circ} \mathrm{C}$ in YPD liquid media, diluted down to an $\mathrm{OD}_{600}=$ 0.1 , and refreshed for $4 \mathrm{~h}$. Cells were then grown with either no treatment (control cells), treatment with FLC alone at $32 \mu \mathrm{g} / \mathrm{ml}$, or FLC at $32 \mu \mathrm{g} / \mathrm{ml}$ and an antioxidant $(10 \mu \mathrm{M}$ PDTC, $1 \mathrm{mM}$ RA, $10 \mathrm{mM} \mathrm{AA}$, or $10 \mathrm{mM} \mathrm{GSH}$ ) for $16 \mathrm{~h}$. Cells were centrifuged at $3000 \mathrm{xg}$ for $2 \mathrm{~min}$ and washed with ice cold PBS $(137 \mathrm{mM} \mathrm{NaCl}, 2.7 \mathrm{mM} \mathrm{KCl}, 10 \mathrm{mM}$

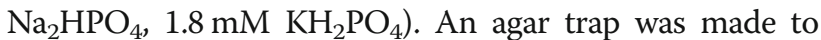
capture yeast cells, by melting $0.8 \%$ agarose on a slide as a thin section. Cells were placed in an agar trap, covered with a coverslip and visualized by Zeiss Axiovert 200 inverted microscope (Carl zeiss, Inc., Thornwood, NY).

\section{Statistical analyses}

For all statistical analyses, the Shapiro Wilk Test was used to test for normality, and afterward, the Bartlett Test was used to test for equality of variance. Since both conditions were met, a multifactorial ANOVA was performed. The Tukey HSD test was used to determine if the relationship between the control group and variable groups were statistically significant.

\section{Supplementary information}

Supplementary information accompanies this paper at https://doi.org/10. 1186/s12866-019-1606-4

Additional file 1. Raw data corresponding to Figures $1 C, 2 C, 3 C$, and $3 D$ that are included in the article.

\section{Abbreviations}

AA: Ascorbic acid; FLC: Fluconazole; GSH: Glutathione; $\mathrm{H}_{2}$ DCFDA: 4-Amino-5methylamino-2',7'-difluorofluorescein diacetate; MIC: Minimum inhibitory concentration; PDTC: Pyrrolidine dithiocarbamate; RA: Retinoic acid; ROS: Reactive oxygen species

\section{Acknowledgments}

We thank Dr. Lukasz Kozubowski for providing the metallothionein mutant strains (originally obtained from Dr. Dennis J. Thiele, Duke University) and for helpful discussions. We thank Furman University Research and Professional Growth (RPG) Fund, the South Carolina NIH-INBRE Faculty Fellows Summer Research Program Fund, and the Furman University Summer Research Fellow awards for supporting this study.

\section{Authors' contributions}

$\mathrm{SC}$ designed the experiments. NHD, MBC, and KN performed the experiments. SC and NHD analyzed the data. SC wrote the manuscript. All the authors contributed to manuscript editing. All authors read and approved the final manuscript.

\section{Funding}

This study was funded by South Carolina NIH INBRE Faculty Fellows Award, Research and Professional Growth (RPG) fund and startup funds at Furman University awarded to SC and Furman University Summer Research Fellow awards granted to MC and KN. The funds were used to purchase all reagents used in this study. In addition, the Summer Research Fellow award provided summer salary to MC and KN and the South Carolina NIH INBRE Faculty Fellows Award provided summer salary to ND and SC. The funding sources have not participated in the design of the study and collection, analysis, and interpretation of data, or in writing the manuscript.

\section{Availability of data and materials}

All data generated during this study are included in this pulished article and in Additional file 1, which contains raw data corresponding to Figures $1 \mathrm{C}$, $2 \mathrm{C}, 3 \mathrm{C}$, and $3 \mathrm{D}$.

\section{Ethics approval and consent to participate}

We confirm that all methods were carried out in proper conformation with appropriate regulations and guidelines. All experimental protocols involving fungal cultures were approved by Furman University. No human or animal subjects were used for this research.

\section{Consent for publication}

Not applicable

\section{Competing interests}

The authors declare that they have no competing interests.

Received: 2 August 2019 Accepted: 29 September 2019

Published online: 06 November 2019

\section{References}

1. Brown SP, Cornforth DM, Mideo N. Evolution of virulence in opportunistic pathogens: generalism, plasticity, and control. Trends Microbiol. 2012;20(7): 336-42.

2. Idnurm A, et al. Deciphering the model pathogenic fungus Cryptococcus neoformans. Nat Rev Microbiol. 2005;3(10):753-64.

3. Park BJ, et al. Estimation of the current global burden of cryptococcal meningitis among persons living with HIV/AIDS. Aids. 2009;23(4):525-30.

4. Assing $\mathrm{K}$, Birgens $\mathrm{H}$, Arendrup M. Cryptococcus neoformans var neoformans resistant to fluconazole in an HIV-negative patient with chronic lymphocytic leukemia. Clin Microbiol Infect. 2003:9(5):441-4.

5. Chen YC, et al. Increasing trend of fluconazole-non-susceptible Cryptococcus neoformans in patients with invasive cryptococcosis: a 12 year longitudinal study. BMC Infect Dis. 2015;15:277.

6. Smith $\mathrm{KD}$, et al. Increased antifungal drug resistance in clinical isolates of Cryptococcus neoformans in Uganda. Antimicrob Agents Chemother. 2015: 59(12):7197-204.

7. Pasko MT, Piscitelli SC, Van Slooten AD. Fluconazole: a new triazole antifungal agent. DICP. 1990;24(9):860-7.

8. Goa KL, Barradell LB. Fluconazole. An update of its pharmacodynamic and pharmacokinetic properties and therapeutic use in major superficial and systemic mycoses in immunocompromised patients. Drugs. 1995: 50(4):658-90

9. $\quad \mathrm{Li} Z$, et al. Fluconazole plus flucytosine is a good alternative therapy for non-HIV and non-transplant-associated cryptococcal meningitis: a retrospective cohort study. Mycoses. 2019;62:686

10. Khan $A A$, et al. Additive potential of combination therapy against cryptococcosis employing a novel amphotericin B and fluconazole loaded dual delivery system. Eur J Pharm Sci. 2018;119:171-8.

11. Revankar SG, et al. Cloning and characterization of the lanosterol 14alphademethylase (ERG11) gene in Cryptococcus neoformans. Biochem Biophys Res Commun. 2004;324(2):719-28. 


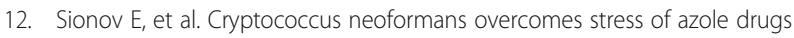
by formation of disomy in specific multiple chromosomes. PLoS Pathog. 2010;6(4):e1000848.

13. Gast CE, et al. Azole resistance in Cryptococcus gattii from the Pacific northwest: investigation of the role of ERG11. Antimicrob Agents Chemother. 2013:57(11):5478-85.

14. Posteraro B, et al. Identification and characterization of a Cryptococcus neoformans ATP binding cassette (ABC) transporter-encoding gene, CnAFR1, involved in the resistance to fluconazole. Mol Microbiol. 2003;47(2):357-71.

15. Cowen LE, et al. Mechanisms of antifungal drug resistance. Cold Spring Harb Perspect Med. 2014;5(7):a019752.

16. Mahl CD, et al. Induction of ROS generation by fluconazole in Candida glabrata: activation of antioxidant enzymes and oxidative DNA damage. Diagn Microbiol Infect Dis. 2015;82(3):203-8.

17. Wang $Y$, et al. Ascorbic acid decreases the antifungal effect of fluconazole in the treatment of candidiasis. Clin Exp Pharmacol Physiol. 2009;36(10):e40-6.

18. Kobayashi $D$, et al. Endogenous reactive oxygen species is an important mediator of miconazole antifungal effect. Antimicrob Agents Chemother. 2002;46(10):3113-7.

19. Peng CA, et al. Fluconazole induces ROS in Cryptococcus neoformans and contributes to DNA damage in vitro. PLoS One. 2018;13(12):e0208471.

20. Winterbourn CC. Reconciling the chemistry and biology of reactive oxygen species. Nat Chem Biol. 2008;4(5):278-86.

21. Angele-Martinez C, Goodman C, Brumaghim J. Metal-mediated DNA damage and cell death: mechanisms, detection methods, and cellular consequences. Metallomics. 2014;6(8):1358-81.

22. Fedorova M, Bollineni RC, Hoffmann R. Protein carbonylation as a major hallmark of oxidative damage: update of analytical strategies. Mass Spectrom Rev. 2014;33(2):79-97.

23. Van Hauwenhuyse F, Fiori A, Van Dijck P. Ascorbic acid inhibition of Candida albicans Hsp90-mediated morphogenesis occurs via the transcriptional regulator Upc2. Eukaryot Cell. 2014;13(10):1278-89.

24. Yang $\mathrm{H}_{\text {, et }}$ al. Structural mechanism of ergosterol regulation by fungal sterol transcription factor Upc2. Nat Commun. 2015;6:6129.

25. Raja MR, et al. A copper hyperaccumulation phenotype correlates with pathogenesis in Cryptococcus neoformans. Metallomics. 2013;5(4):363-71.

26. Zhang $P$, et al. Effects of CTR4 deletion on virulence and stress response in Cryptococcus neoformans. Antonie Van Leeuwenhoek. 2016;109(8):1081-90.

27. Jiang $N$, et al. A copper-responsive factor gene CUF1 is required for copper induction of laccase in Cryptococcus neoformans. FEMS Microbiol Lett. 2009;296(1):84-90.

28. Husain N, Mahmood R. Copper (II) generates ROS and RNS, impairs antioxidant system and damages membrane and DNA in human blood cells. Environ Sci Pollut Res Int. 2019:26:20654.

29. Ding $C$, et al. The copper regulon of the human fungal pathogen Cryptococcus neoformans H99. Mol Microbiol. 2011;81(6):1560-76.

30. Ding C, et al. Cryptococcus neoformans copper detoxification machinery is critical for fungal virulence. Cell Host Microbe. 2013;13(3):265-76.

31. Niedzwiecka K, et al. Glutathione may have implications in the design of $3-$ bromopyruvate treatment protocols for both fungal and algal infections as well as multiple myeloma. Oncotarget. 2016;7(40):65614-26.

32. Altamirano $\mathrm{S}$, et al. Fluconazole-induced ploidy change in Cryptococcus neoformans results from the uncoupling of cell growth and nuclear division. mSphere. 2017;2(3):e00205-17. https://doi.org/10.1128/mSphere. 00205-1.

33. Vasak M. Advances in metallothionein structure and functions. J Trace Elem Med Biol. 2005;19(1):13-7.

34. Upadhya R, et al. Global transcriptome profile of Cryptococcus neoformans during exposure to hydrogen peroxide induced oxidative stress. PLoS One. 2013;8(1):e55110

35. Mesa-Arango AC, et al. The production of reactive oxygen species is a universal action mechanism of amphotericin B against pathogenic yeasts and contributes to the fungicidal effect of this drug. Antimicrob Agents Chemother. 2014;58(11):6627-38.

36. Ferreira GF, et al. The role of oxidative and nitrosative bursts caused by azoles and amphotericin B against the fungal pathogen Cryptococcus gattii. J Antimicrob Chemother. 2013;68(8):1801-11.

37. Zhu BZ, Carr AC, Frei B. Pyrrolidine dithiocarbamate is a potent antioxidant against hypochlorous acid-induced protein damage. FEBS Lett. 2002;532(1-2):80-4.
38. Ahlemeyer $B$, et al. Retinoic acid reduces apoptosis and oxidative stress by preservation of SOD protein level. Free Radic Biol Med. 2001:30(10):1067-77.

39. Zyracka E, et al. Ascorbate abolishes auxotrophy caused by the lack of superoxide dismutase in Saccharomyces cerevisiae. Yeast can be a biosensor for antioxidants. J Biotechnol. 2005;115(3):271-8.

40. Boatright WL. Oxygen dependency of one-electron reactions generating ascorbate radicals and hydrogen peroxide from ascorbic acid. Food Chem. 2016;196:1361-7.

41. Munro D, Treberg JR. A radical shift in perspective: mitochondria as regulators of reactive oxygen species. J Exp Biol. 2017;220(Pt 7):1170-80.

42. Conrad $\mathrm{M}$, et al. Regulation of lipid peroxidation and ferroptosis in diverse species. Genes Dev. 2018;32(9-10):602-19.

43. Linares $\mathrm{CE}$, et al. Fluconazole and amphotericin-B resistance are associated with increased catalase and superoxide dismutase activity in Candida albicans and Candida dubliniensis. Rev Soc Bras Med Trop. 2013;46(6):752-8.

\section{Publisher's Note}

Springer Nature remains neutral with regard to jurisdictional claims in published maps and institutional affiliations.

Ready to submit your research? Choose BMC and benefit from:

- fast, convenient online submission

- thorough peer review by experienced researchers in your field

- rapid publication on acceptance

- support for research data, including large and complex data types

- gold Open Access which fosters wider collaboration and increased citations

- maximum visibility for your research: over $100 \mathrm{M}$ website views per year

At BMC, research is always in progress.

Learn more biomedcentral.com/submissions 